\title{
Obstacles limitant la détection et la prise en charge de l'HPP par les prestataires de soins de santé à Madagascar
}

Breakthrough RESEARCH

Follow this and additional works at: https://knowledgecommons.popcouncil.org/departments_sbsr-rh

Part of the Health Communication Commons, and the Public Health Commons

How does access to this work benefit you? Let us know!

\section{Recommended Citation}

Breakthrough RESEARCH. 2020. "Obstacles limitant la détection et la prise en charge de l'HPP par les prestataires de soins de santé à Madagascar," Sommaire de recherche. Washington, DC: Population Council. 


\section{Résultats}

\section{Aperçu général}

Les prestataires travaillent dans un contexte de pénurie. Bien que bon nombre d'entre eux fassent preuve d'une grande ingéniosité pour relever les défis structurels qui nuisent à leur travail, surmonter ces défis est une préoccupation de premier plan et qui risque d'imposer la largeur de bande cognitive des prestataires de soins, ce qui affecte leur capacité à réagir et à gérer I'HPP complexe et autres complications obstétricales. Alors que les prestataires du monde entier sont souvent aux prises avec une charge de travail excessive, les conditions décrites par ces prestataires malgaches donnent à penser qu'une partie importante de leur bande passante cognitive ${ }^{4}$, ou énergie mentale, est occupée à chercher des moyens de surmonter des problèmes structurels fondamentaux. Ces défis vont de la rupture des stocks de produits et de médicaments et du manque d'électricité dans les établissements aux pressions exercées pour faire payer les services aux clients à faible revenu ou pour convaincre les membres de la famille de parcourir de longues distances pour obtenir des soins de qualité. Comme l'a fait remarquer une sage-femme : " Il y a tant de choses à faire et je ne dors pas assez. »

\section{Détection et prise en charge de l'HPP}

Première observation : En raison de la faible prévalence perçue de I'HPP, le risque d'autres complications préoccupe davantage les prestataires, ce qui les amène à sous-estimer l'importance de se conformer strictement aux mesures préventives. Les prestataires ont fréquemment mentionné qu'ils étaient à l'affût de signes d'un travail prolongé pour se référer à un niveau plus élevé. Étant donné la faible probabilité perçue de I'HPP par rapport à d'autres complications, les prestataires de soins sous-estiment peut-être l'importance de se conformer strictement aux mesures préventives de I'HPP, comme l'administration d'ocytocine après la naissance. De nombreux prestataires ont cité la GATPA, en particulier l'injection d'ocytocine après l'accouchement, comme procédure standard. Cependant, nos résultats suggèrent une application incohérente de la GATPA. Lorsque le risque d'HPP est considéré comme faible, des compromis comme les coûts pour les patientes peuvent amener les prestataires à suivre de façon sélective des pratiques exemplaires comme l'administration d'ocytocine, souvent sur la base d'une heuristique défectueuse pour le risque.

Deuxième observation : Il est difficile de savoir si l'ocytocine est administrée dans la minute qui suit la naissance du bébé, et la différence entre l'administration d'ocytocine dans la minute qui suit et peu de temps après n'est pas évidente, ce qui amène les prestataires à appliquer l'ocytocine plus tard sans savoir que c'est le cas. Lors de la description du processus d'accouchement, aucun prestataire n'a spécifiquement mentionné la bonne pratique consistant à administrer l'ocytocine dans la minute qui suit la naissance, mais les diverses tâches liées aux soins immédiats du nouveau-né avant la GATPA suggèrent que ce délai est en pratique beaucoup plus long qu'une minute. Avec peu ou pas d'exposition aux cas d'HPP, il n'y a pas de conséquences saillantes pour les prestataires de soins de santé de comparer ce qui se passe lorsqu'ils administrent de l'ocytocine en moins d'une minute plutôt que plus tard. Sans cette rétroaction, les prestataires ignorent qu'ils doivent reconsidérer leur comportement.

Troisième observation : Les femmes présentant une HPP sont peu nombreuses. Par conséquent, I'hypothèse par défaut des prestataires est qu'il n'y aura pas d'HPP. Ils n'évaluent donc pas systématiquement les pertes sanguines jusqu'à ce qu'ils aient des indices visibles leur indiquant le contraire, ce qui retarde la détection et la prise en charge. La plupart des prestataires ont vu peu de cas d'HPP dans la pratique, ce qui les amène à supposer qu'il n'y aura pas d'HPP. Ils s'attendent également à ce que des signes clairs et évidents leur indiquent si une femme souffre d'HPP. Cependant, les prestataires de soins ont souvent cité les défis liés à la mesure de la perte de sang ou ne semblaient pas envisager de mesurer systématiquement la perte de sang du tout : « Nous l'évaluons simplement à l'œil. II n'y a rien à utiliser pour la mesurer correctement. » Au lieu d'évaluer systématiquement les pertes sanguines, les prestataires de soins se fient à une quantité excessive de sang sur du tissu blanc comme indice visuel de la présence d'HPP, ce qui peut potentiellement retarder la détection.

Quatrième observation : Le modèle mental $\left.\right|^{5}$ des prestataires de soins en ce qui a trait à l'hémorragie est celui d'une perte de sang rapide et extrême. Par conséquent, les prestataires de soins peuvent renvoyer chez elles les patientes ayant une hémorragie lente et continue sans leur diagnostiquer une HPP. Les expériences et les attentes en matière de saignements décrites par les prestataires de soins reflètent le modèle mental selon lequel I'HPP nécessite une perte de sang rapide et extrême, et lorsqu'on les a interrogés, presque personne ne pouvait se rappeler avoir vu ou entendu parler d'un cas où le saignement était lent mais continu. Dans un contexte où les patientes et les familles peuvent être impatients de quitter l'établissement de soins et où les prestataires subissent des pressions pour libérer de l'espace et des lits limités, les prestataires peuvent supposer que les patientes vont bien après un accouchement simple et ainsi les renvoyer chez elles trop vite. Cette tendance à supposer que l'HPP ne peut être présente entraîne des retards dans la détection et la prise en charge rapide de I'HPP et pourrait entraîner des cas manqués dans la collectivité.

Cinquième observation : Les prestataires peuvent se fier aux membres de la famille ou aux patientes pour leur dire s'il y a un saignement plutôt que de surveiller ceci eux-mêmes, surtout si ils travaillent seuls ou si l'accouchement a lieu tard le soir, ce qui peut retarder le dépistage. L'absence d'une surveillance post-partum cohérente peut entraîner des retards dans la détection de l'HPP. Un superviseur à Tana a fait remarquer au sujet des OEC : " La patiente est là et ils [le personnel] vont dormir un certain temps. Ils reviennent, puis ils découvrent [PPH]. » Lorsque les 
prestataires sont occupés et incapables de vérifier régulièrement les patientes après l'accouchement ou qu'ils croient que ce n'est pas nécessaire, ils peuvent se fier aux patientes ou à leur famille pour leur dire s'il y a du saignement. Bien qu'elles reconnaissent le risque d'auto-évaluation, les sages-femmes épuisées risquent de vouloir se convaincre que la probabilité de complications est faible ou, si elles travaillent dans une situation de pénurie ${ }^{3}$, elles risquent de se concentrer sur les risques les plus importants-fort probablement les femmes en travail, plutôt que la femme qui a déjà accouché.

Sixième Observation : Malgré une formation en gestion de l'HPP, le petit nombre de cas qu'un prestataire de pourrait être amené à traiter rend facile d'oublier ce qu'il faut faire, surtout que la plupart des établissements de santé n'ont pas de rappels visuels des procédures cliniques faciles d'accès (ou peut-être que les prestataires ne les comprennent pas intuitivement) ou personne à qui poser des questions sur-le-champ. La plupart des prestataires de services interrogés avaient vu très peu de cas d'HPP en dehors de leur formation et avaient de la difficulté à décrire les mesures à prendre pour gérer un cas. Étant donné le faible nombre de cas qu'ils ont pris en charge et la variation des protocoles de traitement selon la source de l'HPP, il est peu probable que les prestataires se rappellent ce qu'il faut faire en présence d'un tel cas sans aide quelconque. Bien que plusieurs médecins aient déclaré avoir consulté des livres, dans une situation où les saignements sont abondants et où le prestataire est seul, il est difficile d'imaginer comment un prestataire pourrait facilement se référer à un livre pour savoir comment procéder. Par conséquent, il est probable que les prestataires suivent simplement leur intuition quant à la marche à suivre. De même, les prestataires ruraux sont susceptibles de travailler seuls sans supervision directe, ce qui les oblige à prendre leurs propres décisions.

\section{Septième observation : Il existe un décalage entre les} pratiques sur lesquelles les prestataires sont formés et ce qui est faisable dans les établissements, ce qui laisse aux prestataires le soin de chercher les moyens d'adapter les meilleures pratiques en fonction de leur propre expérience ou de ce que d'autres membres de la formation sanitaire leur disent de faire. Les prestataires reçoivent une formation sur les meilleures pratiques de gestion de l'HPP, mais ont peu d'expérience dans leur application pratique. Les prestataires ont décrit la nécessité d'adapter leur pratique aux contraintes d'équipement des établissements ruraux ou aux habitudes des autres membres de l'établissement de santé plus âgés, même s'ils ont été formés moins récemment. Une sagefemme stagiaire a décrit : " II y a des moments où vous avez l'impression de maîtriser les choses, mais quand vous pensez au Major ou au Chef et qu'ils vous disent qu'ils sont des anciens, qu'ils ont l'expérience et qu'il faut faire comme eux, [même] s'il y a quelque chose qui cloche $»$.

Huitième observation : Les produits et médicaments essentiels pour gérer I'HPP ne sont pas disponibles dans les établissements. Par conséquent, les prestataires lisent les brochures des médicaments qu'ils ont en main pour déterminer ce qu'ils pourraient potentiellement utiliser. Par exemple, certains prestataires utilisent la vitamine $\mathrm{K} 1$ pour la prise en charge de I'HPP puisque l'une des indications médicales mentionne la coagulation sanguine (quoique pour les nouveau-nés). Dans des situations difficiles, face à l'incertitude, les prestataires peuvent se fier aux documents disponibles pour déterminer le traitement de certaines conditions, lequel peut être mal aligné avec les meilleures pratiques. La fréquence à laquelle la vitamine K1 a été mentionnée comme méthode de gestion de l'HPP suggère que les stagiaires et peut-être d'autres prestataires utilisent les brochures médicales pour voir lesquels des médicaments à leur disposition pourraient être utilisés pour différents cas auxquels ils sont confrontés. L'acide tranexamique n'était pas un traitement dont les prestataires avaient entendu parler.

\section{Autres observations}

Dans un pays où la plupart des naissances n'ont pas lieu dans un établissement de santé, la décision des femmes d'accoucher à domicile et la détection et l'orientation des cas de l'HPP pendant les accouchements à domicile ont une incidence sur la mesure dans laquelle le comportement des prestataires en formation sanitaire peut avoir des conséquences en termes de mortalité. Bien que ces autres comportements n'aient pas été pleinement explorés dans cette étude, nous notons ci-dessous quelques idées émergentes liées aux expériences des femmes post-partum et des accoucheuses traditionnelles. Nous recommandons que des recherches supplémentaires soient faites pour tirer parti de ces résultats préliminaires de manière plus concluante.

Première observation : L'accouchement dans un établissement de santé implique de nombreux compromis et, dans un contexte de ressources limitées, l'accouchement à domicile peut constituer le choix le plus rationnel pour de nombreuses femmes.

Deuxième observation : L'accouchement dans un établissement nécessite une planification préalable et les femmes ne planifient pas de cette façon, ce qui peut être aggravé par la crainte de ce qui pourrait arriver si d'autres personnes connaissent la date de l'accouchement.

Troisième observation : Les établissements et l'équipement médicaux peuvent sembler « étrangers » et intimidants pour les femmes, ce qui peut être aggravé par un sentiment de honte quant à leur statut socioéconomique ou par la crainte de mauvais traitements.

Quatrième observation : Dans un contexte où l'accès aux soins de santé en formation sanitaire est limité, des récits culturels ont été élaborés expliquant les complications d'une manière qui remet en question l'utilité d'accoucher dans un établissement puisque ces problèmes n'y seraient pas traités efficacement.

Cinquième observation : Les accoucheuses traditionnelles se concentrent principalement sur l'accouchement d'un bébé en bonne santé et peuvent quitter le domicile de la femme peu de temps après l'accouchement ou ne pas noter un saignement immédiatement pendant qu'elles s'occupent de l'enfant, ce qui peut entraîner un retard dans la détection de I'HPP. 
Sixième observation : Les accoucheuses traditionnelles ne s'assurent pas toujours de l'intégralité du placenta et il en résulte souvent des saignements. Les accoucheuses traditionnelles ne se sentent pas concernées par les saignements qui ne sont pas extrêmes et peuvent ainsi les attribuer à un processus normal de « nettoyage».

Septième observation : La résistance de la femme ou de sa famille à être référée, la confiance excessive en sa capacité de gérer les complications, la crainte des répercussions ou I'incapacité de trouver un moyen de transport en temps opportun peuvent faire en sorte que les accoucheuses traditionnelles essaient de gérer elles-mêmes les cas d'HPP même si elles savent qu'elles devraient référer la femme à un hôpital.

Huitième observation : Le risque d'HPP n'est pas une préoccupation première pour les accoucheuses traditionnelles et I'HPP ne présente souvent pas de signes précurseurs. Par conséquent, les accoucheuses traditionnelles attendent d'être aux prises avec une complication qu'elles ne peuvent pas gérer avant de décider de référer la femme à l'hôpital, ce qui entraîne des retards et des difficultés.

\section{Conclusion}

Ensemble, ces observations soulignent la nécessité de trouver des solutions innovantes pour s'attaquer aux obstacles comportementaux auxquels sont confrontés les prestataires de services lorsqu'ils doivent se conformer aux meilleures pratiques de détection et de gestion de I'HPP, compte tenu surtout des conditions difficiles dans lesquelles ils travaillent. Concevoir pour changer ou s'adapter à des éléments spécifiques dans le contexte des prestataires peut aider à éliminer ou à diminuer l'effet de ces obstacles comportementaux. Par exemple, fournir un soutien en temps réel sur la façon de gérer les complications peu fréquentes en utilisant des supports visuels améliorés ou en offrant un soutien à distance comme prolongement du mentorat pourrait être plus efficace que des efforts de formation qui sont loin du moment critique et non applicables dans la pratique à leur contexte de travail.

Les processus de cocréation en collaboration et les tests itératifs garantiront que les nouvelles interventions soient les mieux positionnées pour avoir un impact et soient spécifiques et adaptées aux besoins des prestataires et des femmes en travail. Cette approche de conception d'intervention a déjà été utilisée avec succès pour développer des solutions liées au comportement des prestataires, comme pour améliorer les soins maternels respectueux en Zambie et le dépistage et le traitement du paludisme au Nigeria, et pourrait être très prometteuse pour améliorer la qualité des soins à apporter en cas d'HPP à Madagascar.

\section{Références}

1. Say, L. et al. 2014. "Global causes of maternal death: a WHO systematic analysis," Lancet Global Health 2(6): e323-e333. doi:10.1016/S2214109X(14)70227-X

2. Rakotozanany, B. et al. 2019. "Maternal mortality related to postpartum hemorrhage: a case-control study at the Befelatanana maternity of Madagascar," International Journal of Reproduction, Contraception, Obstetrics and Gynecology 8(1): 121-126. doi: 10.18203/2320-1770. ijrcog20185406

3. UNICEF. 2014. State of the World's Children 2015. Geneva: UNICEF.

4. Mullainathan, S., E. Shafir. 2013. Scarcity: Why Having Too Little Means So Much. New York: Macmillan.

5. Norman, D.A. 1983. "Some observations on mental models." In Dedre Gentner, Albert L. Stevens (eds) Mental Models. New York: Psychology Press, $15-22$

Ce travail s'inscrit dans le cadre du partenariat APPHC (I'Avancement des Soins de l'Hémorragie de Post-Partum) soutenu par I'USAID et dirigé par le projet Breakthrough RESEARCH et le projet HEARD (Projet d'évaluation de santé et de développement de la recherche appliquée). Le partenariat APPHC génère et teste des solutions pour surmonter les principaux obstacles à la mise en œuvre de la prévention et du traitement de l'HPP et contribue à la mise en œuvre efficace d'interventions, de stratégies et d'innovations pour I'HPP à Madagascar et au Malawi.

Remerciements : Ce sommaire de recherche décrit le travail mené par des idées42 dans le cadre de Breakthrough RESEARCH en collaboration avec ACCESS et les bureaux de santé des districts de Manakaran et de Vohipeno. Nous sommes également reconnaissants de l'appui de toutes les sagesfemmes, femmes et autres participantes aux entrevues qui ont accepté de nous parler durant ce travail.

Citation proposée : Breakthrough RESEARCH. 2020. “Obstacles limitant la détection et la prise en charge de l'HPP par les prestataires de soins de santé à Madagascar," Sommaire de recherche. Washington, D.C. : Population Council.

Crédit photo de la page couverture : @Maddie Kau, ideas 42

(C) 2020 The Population Council. Tous les droits sont réservés.
Breakthrough RESEARCH Population Council

4301 Connecticut Ave., NW | Suite 280

Washington, DC 20008

+12022379400

breakthroughactionandresearch.org BreakthroughResearch@popcouncil.org
USAID's Health Evaluation and Applied Research Development URC

5404 Wisconsin Ave | Suite 800

Chevy Chase, MD 20815

www.heardproject.org

heard@urc-chs.com

\section{USAID}

Le document est rendu possible grâce au soutien généreux du peuple américain à travers l'Agence américaine pour le développement international (USAID) en vertu des termes de I'accords des coopération du Breakthrough RESEARCH ( $n^{\circ}$ AID-OAA-A-17-00018) et du projet d'évaluation de santé et de développement de la recherche appliquée d'USAID (HEARD) (AID-OAA-A-17-00002). Le contenu de ce document relève de la responsabilité exclusive de Breakthrough RESEARCH, du Population Council et de l'University Research Co., LLC (URC) et ne reflète pas nécessairement les positions de I'USAID ou du gouvernement des États-Unis.
USAID'S HEALTH EVALUATION AND APPLIED RESEARCH DEVELOPMENT (HEARD) PROJECT

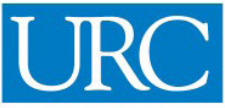

Le projet HEARD de I'USAID s'appuie sur un partenariat mondial de plus de 30 institutions pour générer, synthétiser et utiliser des données probantes afin d'améliorer la mise en œuvre des politiques et des programmes liés aux domaines prioritaires de I'USAID, et essentiels pour améliorer la santé et le développement dans les pays à faibles et moyens revenus.

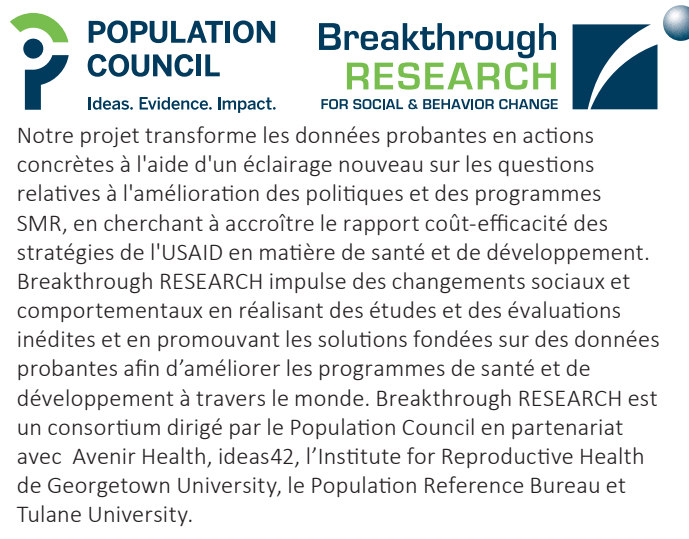

Notre projet transforme les données probantes en actions concrètes à l'aide d'un éclairage nouveau sur les questions relatives à l'amélioration des politiques et des programmes stratégies de l'USAID en matière de santé et de développement. inédites et en promouvant les solutions fondées sur des données probantes afin d'améliorer les programmes de santé et de developpement à travers le monde. Breakthrough RESEARCH est un consortium dirigé par le Population Council en partenariat de Georgetown University, le Population Reference Bureau et Tulane University. 